MOTOR NEURON DISEASE

\section{Synaptotagmin 13 - the key to cell resilience in motor neuron disease?}

The vesicular trafficking protein synaptotagmin 13 (SYT13) protects neurons from degeneration in motor neuron disease, according to a new study. The finding suggests a therapeutic approach that was successfully tested in mice.

In motor neuron diseases, such as amyotrophic lateral sclerosis (ALS) and spinal muscular atrophy (SMA), some populations of motor neurons are resilient to degeneration. In particular, neurons involved in eye movements, such as oculomotor neurons, remain functional into advanced disease stages. Eva Hedlund, who led the new work with Stefania Corti, studies the transcriptional profiles of these resilient neurons to see what makes them different.

"We have a long-standing interest in unravelling mechanisms that underlie the differential vulnerability among motor neuron subpopulations, as we believe this can reveal mechanisms that can be exploited for therapeutic purposes," Hedlund explains.

In previous work, Hedlund, Corti and colleagues identified insulin-like growth factor 2 (IFG2) as a protective factor. The starting point for the new study was a bioinformatics analysis that showed enrichment of SYT13 in oculomotor neurons relative to spinal motor neurons in rodents.

In light of this finding, the researchers analysed SYT13 expression in tissue from humans with ALS. As in rodents, SYT13 expression was upregulated in neurons that remained resilient into end-stage disease. Furthermore, in vitro experiments showed that overexpression of SYT13 in neurons from patients with motor neuron disease protected these cells from degeneration.

The teams tested the therapeutic potential of their findings with gene therapy in mouse models of motor neuron disease. "Syt13 gene therapy prolonged the lives of transgenic ALS mice by $14 \%$ and of SMA mice by $50 \%$ by preserving motor neurons, delaying muscle denervation and reducing endoplasmic reticulum stress," says Corti.

Owing to the complexity of motor neuron disease pathogenesis, the researchers think that targeting the expression of one resilience factor will be insufficient. "We hypothesize that a combination of different oculomotor neuron resilience factors, such as SYT13 and IGF2, could be more beneficial than a single-gene approach," explain Corti and Hedlund. "We are therefore now evaluating a combination therapy using these two genes in transgenic ALS mice." mice by $14 \%$ and of SMA mice by $50 \%$

\title{
Brain and blood biomarkers of chronic traumatic encephalopathy
}

Repetitive mild traumatic brain injury (mTBI) results in tau accumulation that is detectable with PET imaging, according to a new study published in Molecular Psychiatry. The findings raise the possibility that chronic traumatic encephalopathy (CTE) could be diagnosed during life.

Individuals who experience multiple mTBls, such as those caused by shockwaves from blast explosions, can develop memory loss and alterations in mood or personality years after the initial injury. These symptoms can be a sign of the neurodegenerative disease CTE, which is characterized by aggregation of hyperphosphorylated tau in the brain. Currently, CTE can only be confirmed post mortem, but the aim of the new study, led by Gregory Elder and Sam Gandy, was to develop biomarkers to detect CTE in

living individuals and perhaps guide therapeutic intervention.

Excess ligand

retention was

observed in

five of the

veterans

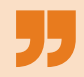

"There are many young, otherwisehealthy military veterans who have suffered blast-related TBIs, some of them years in the past, who either aren't getting better or, in some cases, are getting worse," notes Elder. "We don't know why or how to identify those at greatest risk."

The researchers exposed rats to $\mathrm{mTBI}$ once per day for three consecutive days and analysed brain samples taken at 6 and 10 months after the last injury. The level of phosphorylated tau was higher in mTBIexposed rats than in control rats at both timepoints, showing that tau pathology can be detected long after injury.

The researchers also performed brain imaging with the tau PET ligand ${ }^{18} \mathrm{~F}-\mathrm{AV} 1451$ in ten veterans with histories of repeated blast exposure and symptoms of CTE and seven healthy controls. Excess ligand retention was observed in five of the veterans and none of the controls, suggesting that this approach can identify blast-induced tau pathology in humans. The pattern of ligand retention in the veterans resembled the post-mortem tau pathology usually seen in individuals with CTE.

The researchers also measured blood levels of neurofilament light chain (NfL), which has been used as a biomarker of neurodegeneration, in the veterans and controls. No difference was observed between the two groups, but among the veterans, excess ${ }^{18} \mathrm{~F}-\mathrm{AV} 1451$ retention was associated with higher NfL levels. "Next we are keen to see whether ${ }^{18} \mathrm{~F}-\mathrm{AV} 1451$ and/or NfL can be used to predict which patients benefit from a new drug that relieves symptoms in blast-exposed rats," concludes Gandy.

Sarah Lemprière

ORIGINAL ARTICLE Dickstein, D. L. et al. Brain and blood biomarkers of tauopathy and neuronal injury in humans and rats with neurobehavioural syndromes following blast exposure. Molecular Psychiatry https://doi.org/10.1038/s41380-0200674-z (2020) 